\title{
Study on Design and Production of Augmented Reality Work Integrated with Shadow Art Element
}

\author{
Yuan Yang \\ Sino-Korea Multimedia Design Institute \\ Shanghai University of Engineering Science \\ Shanghai, China
}

\author{
Yingzhou Zhu \\ Sino-Korea Multimedia Design Institute \\ Shanghai University of Engineering Science \\ Shanghai, China
}

\author{
Chaoqun Sui \\ Sino-Korea Multimedia Design Institute \\ Shanghai University of Engineering Science \\ Shanghai, China
}

\begin{abstract}
This paper analyzes and studies the digital media design work produced based on the augmented reality technology. The work applies the shadow play to show the design of role, scene and action. The production process includes both the image production with computer software and the design and production of the interactive exhibition part of the work for the purpose of applying the representative element of the traditional Chinese shadow art to the augmented reality work in a reasonable and effective manner.
\end{abstract}

\section{Keywords -shadow art; augmented reality; shadow element}

\section{ANALYSIS ON DESIGN CONTENT AND APPLIED TECHNOLOGY}

The traditional Chinese shadow play, with a long history, is an art form characterized by folk and regional culture. As a traditional folk art, shadow art reflected in many paper cutting, New Year pictures and carving builds vivid and humorous roles and scene modeling. All figures generated by the shadow have allegoric shape, geometric symbol, simple color combination and proper separation of light and shadow. Shadow art integrates several design languages like visual communication, apparel modeling, real-time interaction and performance. Digital technology brings convenience for people in the field of life whereas many traditional art forms die out. Today digital production technology changes people's living and entertainment manners and redesigns all aspects in daily study, entertainment and life. These increase the design works of traditional culture, folk-custom and art element, presenting a new understanding and interpretation of the traditional art element to some degree while enriching the content and strengthening the fun of interaction.

Augmented reality is a technology via which humans feel the superposition of analog simulation made with digital media technology in real life so as to create the sense experience transcending the reality for the audience. Augmented reality technology is increasingly applied in many areas including business, entertainment and education. Different from general digital technology which demonstrates digital effect with the help of computer software production, it superposes and composites the virtual and reality in the display screen to crease a brand new visual experience for the audience. "Concerto Timer" is a work made with augmented reality launched by Haagen-Dazs. After users download such software and scan the ice cream cover, a figure playing violin will shown on the screen of the mobile phone. This design not only presents beautiful pictures and combines a two-minute sound effect background. The entire work is designed to remind the customers of tasting the ice cream in the first two minutes after being taken out of the refrigerator. By this means, Haagen-Dazs reminds the customers by making the boring waiting time elegant and interesting.

\section{DESIGN BACKGROUND AND PRODUCTION PROCESS}

Shadow art is an intangible cultural heritage of China and many animation works apply such element. This paper analyzes and explains how to reflect the e-book made with 3D augmented reality with traditional art element. The e-book is designed based on historical events of China, the story of which is visually reflected by shadow element. It presents the plane and monotonous story vividly with 3D animation through the carrier of multi-media for the audience. The viewers may appreciate the artistic charm of traditional shadow play while learning cultural knowledge.

The e-book titled Fantastic Double-Sword Story is compiled based on the story that Chen Sheng and Wu Guang staged an uprising at the end of the Qin Dynasty (BC 209). The work is highlighted by the visual effect design, especially the design and production reflecting traditional shadow art element in such aspects as role, scene and animation. The design and production of plane figures like role and scene are made with 2D plan figure design software based on which the $3 \mathrm{D}$ role, scene model, animation and 3D special effect will be produced. In the process, 3D plug-ins including RealFlow, Pf Particle Flow, Ray Fire and Vray need to be used. By means of the AR technology in Unity3D software, lively story images can be generated with the pictures produced by the story line, linked 
as the developmental sequence of the story and finally presented by playing picture scroll. The self-made equipment used in displaying the work is designed as the style of scroll. Viewers may rotate the scroll to identify each picture and form a rich and complete story to interactively demonstrate the work.

\section{PRIMARY LINKS OF PRODUCTION}

\section{A. Role Design and Production}

In accordance with the story setting, all characters in the play are male, most generals and soldiers. To be light, thin, transparent and bright, traditional shadow figures are primarily made with animal leather and hollow carving technique. The purpose of applying computer software in designing and producing role images is to restore the features of the models made with primal process. In order to embody the hollow carving process, the roles are particularly rendered to show the process effect of intaglio. In addition, for the sake of highlighting the difference between the two generals in the story, their faces are colored in black and white, respectively. In the process of design, considering that the e-book made with augmented reality will be principally read by children, in order to better stimulate the interest of children in reading, the role images need to be cartoonish. As a result, a body height three to four times of the head height is designed to make the roles look more amiable and the proportion of eyes on the face exaggerated to increase many child interests. In terms of color rendering, the color matching of high contrast, low brightness and low purity is applied to create a sense of primitive simplicity and thickness as shown in "Fig. 1". Furthermore, with respect to role modeling and representation process, joints of the role are decomposed and combined as shown in "Fig. 2".

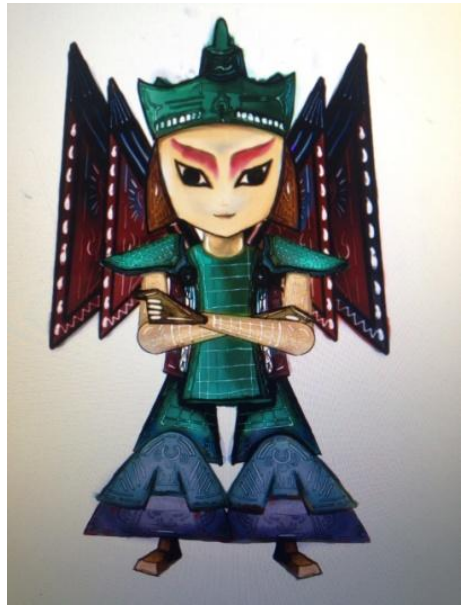

Fig. 1. Role Design Sketch

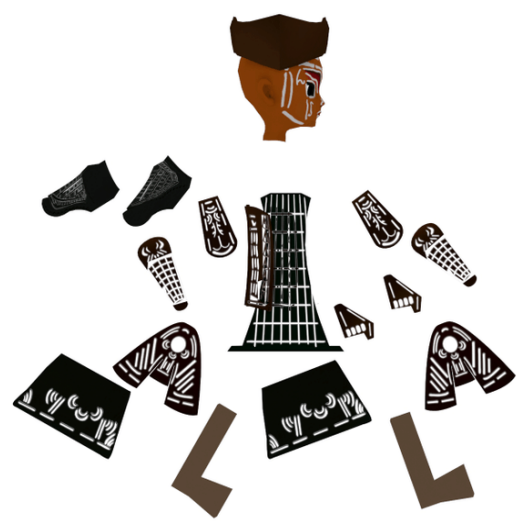

Fig. 2. Design Sketch of Role Decomposition

Upon completion of the plane image design and production of the role, 3D modeling will be conducted for the role with 3dsMax software. Cubic modeling is more convenient for skeleton binding and animation production at the later stage of the role. For the effect of role modeling, see "Fig. 3". As role modeling is composed of cubes, the audience will see a plane image when the direction changes, which is closer to the demonstration effect of traditional shadow play. The figures in traditional shadow play are very vibrant in color combination, so rendering of the role map has a strong contrast. Besides, the brightness of the figure map is lowered to complete the $3 \mathrm{D}$ effect of the roles.

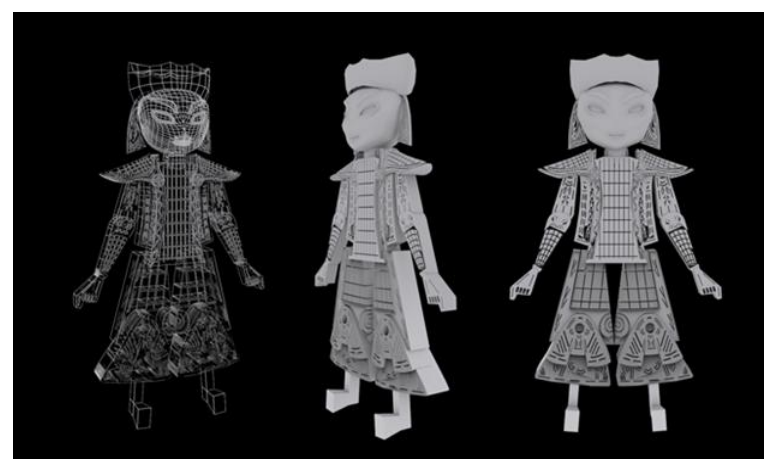

Fig. 3. 3D Model Effect of Role

\section{B. Scene Design and Production}

The scene is designed closed to the artistic style of traditional shadow play, which is reflected in the production of scene model and map. The scene elements in the Fantastic Double-Sword Story like arrow tower, war drum and palace, based on the overall design style of the e-book and representative element of traditional shadow figures, refer to traditional shadow images. 3D modeling and map production is carried out on the basis of designing and drawing plane figures. UV spreading and rendering of maps are made after completing the scene modeling with the method of patch modeling. When the scene map is to be drawn, the inherent color and the color and patterns at different parts should be depicted first. "Fig. 4" shows the effect of local map of the 
scene. The color of the scene which should be consistent with the role style also embodies the effect of cartoon style as shown in "Fig. 5".

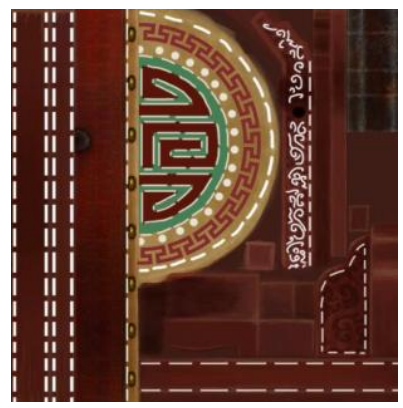

Fig. 4. Drawing of Scene Map

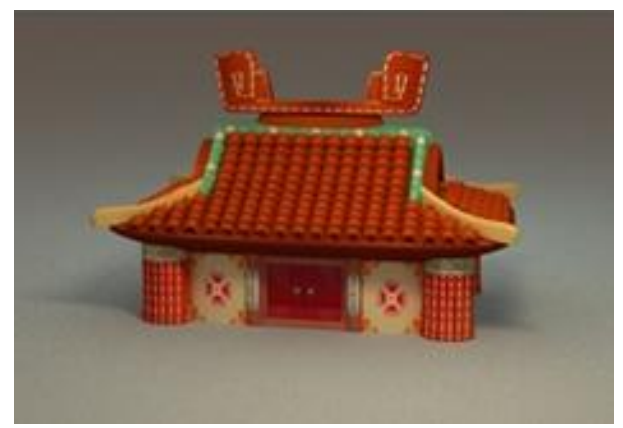

Fig. 5. Production Effect of Palace Model in the Scene

In addition to the production of scene element, the production effect of the opening scene of the story is also very important. The opening image, for attracting the audience, determines the quality of the work directly. Mountain, water, cloud and other natural landscape effect in the opening scene are reflected by shadow element. In the process of production, Illustrator software is used. It sketches the outline of the mountain with pen tool, import these paths to the work path of photograph and finally import the files in 3DsMax software. The path drawn automatically transforms to line transect and then align before being squeezed out. In the course of squeezing, the line transect needs to be inspected again for mountain type mapping when there is no error. In order to better demonstrate the picture effect in the traditional shadow scene, the gradient map in 3DsMax is used. When the effect of the water body at the foot of the mountain is made, water material in the Mental Ray renderer is selected. Value of reflect light and wave are turned up. After rendering, the effect of water waves can be really reflected as shown in "Fig. 6".

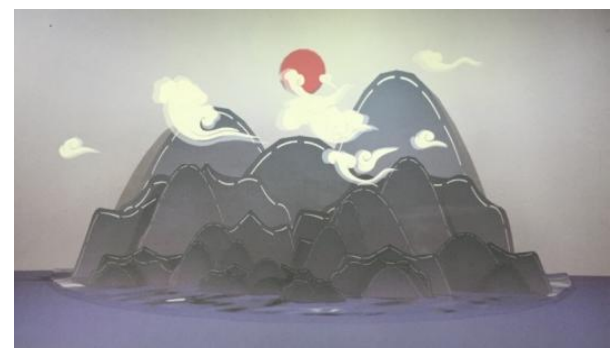

Fig. 6. Map Rendering Effect of Scene
The Fantastic Double-Sword Story selects the wonderful part of the story for 3D rendering production. The effect of water surface and explosion is made with the built-in mental ray of 3dsMax software and the plug-in of Ray Fire is used in blasting animation of the model. The frame of war and smoke in the story are reflected by the plug-in Fume Fx. As there is no keyframe in the smoke produced, it is impossible to import Unity3D in the later stage. Thus a smoke plug-in of Unity3D is used. Vray renderer is used in the scene production of other parts for rendering.

\section{Action Design and Production}

The action of the role is made with the Physique too and CAT skeleton in 3Dsmax. For CAT skeleton, the built-in role model skeleton of 3Dsmax is very proper for producing role animation. "Fig. 7" shows the picture effect after binding skeleton. Physique tool rather than general skin tool is used because it can better modify the weight of the role model point. General skin tools will show bends like human body in the joint of the role after binding the skeleton. However, this is inapplicable to reflecting the action of the shadow role in this work. Therefore, when the skeleton is bound, Physique tool needs to be added for the role model, and then select skeleton and its influence point. Cancel the weight of all influence points and then add a green weight. In this way, the action of the role is able to approach to the effect of the traditional shadow figure that the parts other than the joint don't twist.

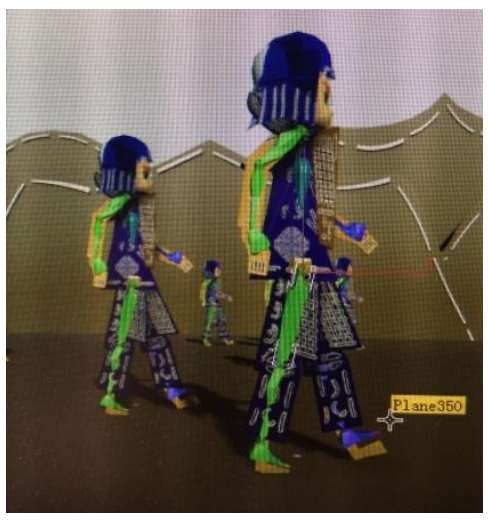

Fig. 7. Role Model Bound with Skeleton

\section{Augmented Reality Production of Unity3D}

After the scene, figure and role action are produced, the part of augmented reality which is made with Unity3D will begin to be produced. First of all, design recognizable patterns and upload them to the designated website for processing. The processed figure can only realize the technical effect of augmented reality after identification. The ability to identify the picture will influence the operation experience of the viewers. The interactive experience of the audience will be significantly impacted if it is hard for them to identify the pictures in operating the equipment. In order to give the viewers a good operation experience, the recognizable pictures and the images to be viewed should be distinguished in a reasonable way. 
Upon uploading the picture, import the 3D file produced to Unity3D and export in the form of FBX file. After importing Unity3D, match the scene picture and recognizable image one by one and adjust the size of the scene. The size of the file in 3dsMax is 100 times of that in Unity3D. Therefore, it is hard to regulate the right size in 3dsMax. When the scene and role are imported, adjust the coordinate position in Unity3D. After the recognizable picture is identified, the augmented reality effect of the image will show in the right position of the screen. If the coordinate of the role is not reset in case of importing, the location of the role will have a large deviation after the file is imported to Unity3D, skeleton binding has to be made for the role by returning to the $3 \mathrm{dsMax}$ software.

\section{E. Production and Demonstration of Interactive Device}

The work is demonstrated with the help of the self-made device and the interaction is realized by computer projection. The picture for identification needs to be fixed with a device. A scroll is also needed for placing the recognizable picture. The location of the scroll also needs to be reinforced. First measure the size of the scroll. The diameter of the scroll handle is 3.5. As the size of internal picture album equals to an A4 paper, the size of the device for fixing has to be larger than A4. Based on the data measured, make a case with a proper size, on which four holes are punched for fixing the scroll. Install the camera at the bottom of the cabinet for shooting recognizable picture. As the camera is installed below, a lightcompensating lamp is also installed for strengthening the identification capability of the camera. At last, the production of interactive device is completed by connecting the computer and TV as shown in "Fig. 8".

When the picture is identified with camera, the image in Unity $3 \mathrm{D}$ always jitters, so the camera and recognizable picture should first be fixed. In the process of fixing, in order to avoid slight jitter, the coordinate code is modified in Unity $3 \mathrm{D}$ to prevent the situation of failure to identify the picture when users have an experience, which also keeps the mystery of camera to make the experience effect more interesting. The scroll painting can be rotated by turning the left and right scrolls in the process of user experience to switch the scene so that the animation of each scene will show on the computer screen as exhibited in "Fig. 9".

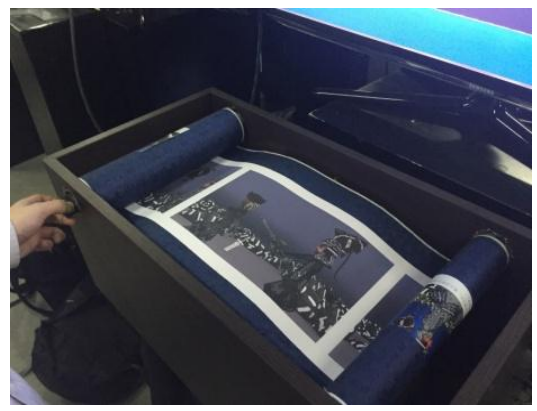

Fig. 8. Interactive Equipment of the Work

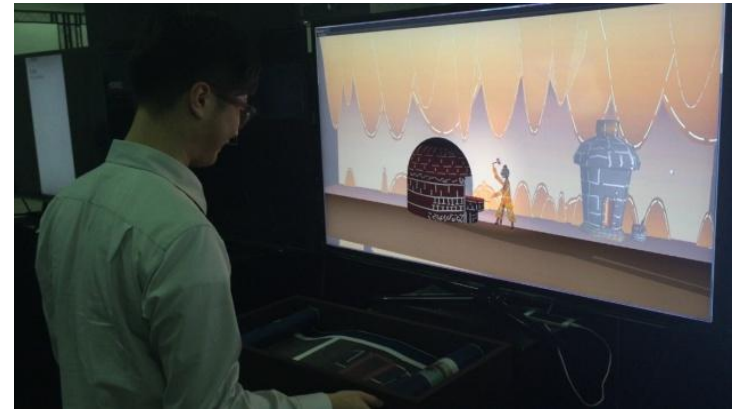

Fig. 9. Demonstration Effect of the Work

\section{CONCLUSION}

The Fantastic Double-Sword Story is expressed by applying traditional Chinese artistic form from story planning to design element. At the point of integrating the traditional and modern culture, a new artistic presentation is formed by combining shadow element and new media art and blending modern scientific technology and aesthetic taste. The e-book designed by integrating traditional Chinese shadow element under the support of augmented reality technology tells the story in another form. Augmented reality technology overturns the traditional expression way so that the shadow play is performed in modern digital artistic form. This combination of modern technology and traditional art, a redefinition and expression of traditional literature and art, presents a new appearance for the audience.

\section{REFERENCES}

[1] Wu Leying. Application and Study of Shadow Art in Animation Design [J]. Popular Literature, Issue 17, 2010.

[2] Yang Fangqi, Luo Weiliang. Reflection on Digital Development of Shadow Play of Shaanxi East Mansion [J]. Value Engineering, Issue 35, 2010.

[3] Zhu Yerong. "Shadow" and "Moving" [D]. Hunan Normal University, 2009.

[4] Jia Tao, Wei Kai. Simple Analysis on Characteristics of Folk Shadow Model Art[J]. Art Panorama, Issue 7, 2007. 